\title{
BioFeedback
}

\section{Addendum: Site-directed mutagenesis using Pfu DNA polymerase and T4 DNA ligase}

The authors (Adereth et al., BioTechniques 38:864-868; June 2005) wish to acknowledge a prior paper (Ali and Steinkasserer; BioTechniques 18:746-750; May 1995) that also relied upon phosphorylation of the first-round PCR products to generate a gene fusion and $27 \mathrm{bp}$ insertional mutants. However, the earlier paper utilized $\mathrm{T} 4$ polynucleotide kinase to in vitro phosphorylate first-round PCR products. Presuming that the earlier method is efficient in creating all the various types of mutations described in the paper, our method has the following advantages:

(i) The T4 polynucleotide kinase step is not required. This is significant cost-savings, especially when a large number of mutations need to be introduced.

(ii) No intermediate heat denaturation or additional enzyme reaction step is required, thus reducing handson time.

(iii) As opposed to the use of T4 polynucleotide kinase, the use of phosphorylated PCR primers ensures efficient phosphorylation of the PCR product. Notably, phosphorylation of one end of the PCR product has the distinct advantage of allowing ligation of the first-round PCR products in the correct orientation, thereby significantly limiting undesirable combinations of the ligated product. We find this feature to be crucial for the correct amplification of difficult templates (e.g., GC-rich templates). (iv) We describe the use of vector sequences to generate first-round PCR products of convenient size for creating gene fusions or mutations along the entire length of a cloned cDNA.

Editor's Note: The manuscript submitted by Adereth et al. was accepted for publication following evaluation by the editors and taking into consideration the recommendations of three independent reviewers. That an aspect of Adereth et al.'s approach which had been previously described was not identified by exhaustive literature searches conducted by the authors is evidence of the fact that details of methods can elude even thorough searches of the literature and we commend the authors for acknowledging the earlier publication. 